\title{
Oficinas de estimulação cognitiva para idosos com demência: uma estratégia de cuidado na enfermagem gerontológica ${ }^{1}$
}

\author{
Priscilla Alfradique de Souza*, Rosimere Ferreira Santana", Selma Petra Chaves Sá"*, \\ Lorena Maria Volkers Robers"*;
}

\section{Resumo}

Objetivamos descrever e analisar a eficácia das oficinas de estimulação cognitiva para idosos com demência pela aplicação de instrumentos básicos de rastreio cognitivo. Para tanto utilizamos a abordagem quantitativa, prospectiva e quase-experimental, aplicada a 11 idosos no período de agosto de 2006 a julho de 2007, nas dependências do Programa de Geriatria e Gerontologia da Universidade Federal Fluminense, Niterói - RJ. Os resultados obtidos demonstram a caracterização do perfil da clientela e o tratamento estatístico dos testes longitudinalmente. Podemos concluir a viabilidade do uso da estratégia no processo de trabalho da enfermeira atuante em gerontologia.

Palavras-chave: Enfermagem geriátrica. Saúde do idoso. Doença de Alzheimer.

\section{Introdução}

Trata-se de um estudo sobre o uso das oficinas de estimulação cognitiva para idoso com demência como estratégia do cuidado de enfermagem gerontológica, tendo como objetivos descrever e analisar a eficácia dessas pela aplicação de instrumentos básicos de rastreio cognitivo.

A necessidade de estudos que apontem perspectivas saudáveis e aplicáveis ao cenário de crescimento populacional e aumento da expectativa de vida, com o consequente incremento das doenças crônicas não transmissíveis, tornou-se um desafio para o cuidado às síndromes

* Bolsista Pibic/CNPq. Graduada e licenciada em Enfermagem pela Escola de Enfermagem Aurora de Afonso Costa Universidade Federal Fluminense.

** Professora Assistente da Universidade Federal Fluminense. Doutora e mestra em Enfermagem pela Universidade Federal do Rio de Janeiro. Especialista em Psicogeriatria pela Universidade Federal do Rio de Janeiro.

*** Professora Titular da Universidade Federal Fluminense. Doutora em Enfermagem pela Universidade Federal do Rio de Janeiro. Mestra em Enfermagem pela Unirio. Coordenadora do projeto "Enfermagem no Programa Interdisciplinar de Geriatria e Gerontologia da Universidade Federal Fluminense".

**** Graduada e licenciada em Enfermagem pela Escola de Enfermagem Aurora de Afonso Costa Universidade Federal Fluminense. Endereço para correspondência: Rua São Sebastião, 78, Bl B, apto 812, Bairro Ingá, Niterói - RJ. CEP 9203-1975. E-mail: lorenarobers@hotmail.com

1 Recorte ampliado do projeto de pesquisa Pibic/CNPq: "Oficina terapêutica para idosos com demência e suporte aos cuidadores: uma proposta de enfermagem".

$\hookrightarrow$ Recebido em dezembro de 2008 - Avaliado em junho de 2009.

$\rightarrow$ doi:10.5335/rbceh.2009.035 
demenciais, já que achados epidemiológicos revelam um aumento da ocorrência exponencial com a idade, pois dobram, aproximadamente, a cada 5,1 anos a partir dos sessenta anos de idade. (BOTTINO; LAKS; BLAY, 2006).

Conceituamos demência como uma síndrome, ou seja, um conjunto de sinais e sintomas característicos decorrentes de uma doença cerebral. Apresenta como padrão cognitivo central o prejuízo da memória e a presença de, pelo menos, mais um dos seguintes transtornos: afasia (prejuízo na linguagem pela ruptura da função cerebral), apraxia (incapacidade de realizar atividades motoras complexas, apesar da capacidade motora intacta), agnosia (falha em reconhecer ou identificar objetos, apesar das funções sensoriais intactas) e em funções executivas de planejamento, organização, sequência e abstração. (BOTTINO; LAKS; BLAY, 2006).

Portanto, as barreiras cognitivas que essa síndrome impõe interferem nas atividades de vida diária do idoso, tornando-o cada vez mais dependente da família e/ ou cuidador formal e informal para a realização das tarefas. (GROISMAN, 2004). Portanto as oficinas de estimulação cognitiva, ou, conforme denominação, "oficinas terapêuticas", visam estimular o idoso à realização de atividades voltadas para a memória e para o exercício das funções cognitivas. Desse modo, permite-se um retardo no desenvolvimento da doença e/ou a produção de formas de adaptação às novas situações vivenciadas. As oficinas com idosos que apresentam síndrome demencial objetiva, acima de tudo, a estimulação das atividades preservadas e a reabilitação das funções cognitivas. (BOTTINO; LAKS; BLAY, 2006; GROISMAN, 2004).

Santana, Santos e Caldas (2005), relatam que, no que tange ao cuidado da enfermagem gerontológica ao atendimento do binômio idoso-cuidador, temos uma concentração de pesquisas na área de atenção aos cuidadores, com estudos sobre demência e enfermagem, abrangendo temas como estresse do cuidador, (CALDAS, 2000; MUÑOZ; ARANCIBIA; PAREDES, 1999; PELTZER; FERNANDES, 1997) e descrições das experiências de cuidado familiar no âmbito domiciliário. (COELHO, 2004; MENDONZA, 1998; SOUZA; PEREIRA; SCATENA, 2002). Porém, não encontramos estudos sobre a atuação da enfermeira, dirigidos direta e especificamente à sua participação nas oficinas de estimulação cognitiva, o que evidencia uma lacuna no conhecimento existente.

$\mathrm{O}$ enfermeiro integrante da equipe multidisciplinar pode contribuir para a otimização das funções cognitivas, minimização dos problemas de comportamento e melhoria do funcionamento global, além de possibilitar uma redução do estresse dos cuidadores e, consequentemente, a prevenção de uma possível institucionalização dessas pessoas. (SANTANA; SANTOS; CALDAS, 2005; BOTTINO; LAKS; BLAY, 2006).

Este estudo, na medida em que descreve e analisa a eficácia de uma proposta de cuidado de enfermagem gerontológico, contribui para o ensino na área de enfermagem e saúde do idoso, além de oferecer subsídios para o desenvolvimento e aprofundamento dos achados em futuras pesquisas. 


\section{Metodologia}

Para elaboração do presente estudo recorremos à abordagem quantitativa de pesquisa, apoiada na necessidade de se compararem os eventos por meio de instrumentos de medida. Dessa forma, classifica-se como uma aplicação ativa de um modelo eficiente, planejado e adequado à parcela da população estudada. Para tanto, escolheram-se o estudo prospectivo, do tipo quase-experimental, por não se pretender o controle das variáveis, e a amostra do tipo não aleatória.

$\mathrm{O}$ estudo foi realizado nas dependências utilizadas pelo projeto "A Enfermagem no Programa de Geriatria e Gerontologia da Universidade Federal Fluminense" (EPIGG/UFF), que está inserido num grande programa de geriatria da universidade em Niterói, Rio de Janeiro. O projeto atende idosos da região Metropolitana 2, que compreende os municípios de Niterói, São Gonçalo, Itaboraí, Silva Jardim, Rio Bonito e Maricá.

\section{Casuística}

Os idosos foram, primeiramente, atendidos na consulta de enfermagem, na qual, ao se identificar alguma suspeita de défice cognitivo, aplicavam-se os instrumentos de rastreio cognitivo (pré-avaliação). Assim, aqueles que apresentavam défice cognitivo, e cujos cuidadores possuíam disponibilidade, eram encaminhados para as oficinas terapêuticas. Dessa forma, foram acompanhados no período de agosto de 2006 a julho de 200711 idosos com síndrome demencial, segundo os critérios diagnósticos recomendados pela Diagnostic and Statistical Manual of Mental Disorders, quarta edição - DSM IV. A partir da pré-avaliação, os idosos encaminhados para a oficina de estimulação foram reavaliados de três em três meses com os mesmos instrumentos de rastreio cognitivo. Assim, foram realizadas uma préavaliação, pós-avaliação 1 , pós-avaliação 2 e pós-avaliação 3 .

\section{Instrumentos de avaliação}

A coleta de dados foi realizada trimestralmente pela aplicação do Mini Exame do Estado Mental (MEEM), do Teste do Relógio, do Teste de Evocação de Palavras, da Escala de Depressão Geriátrica (EDG), da Escala de Atividade Instrumental de Vida Diária (AIVD - Lawton) e da Escala de Atividade de Vida Diária (AVD - Katz), instrumentos mundialmente conhecidos e utilizados na saúde do idoso.

\section{Estratégias de estimulação cognitiva}

As intervenções no grupo de idosos ocorriam semanalmente, às quintasfeiras à tarde, desenvolvidas em dois tempos com duração média de $50 \mathrm{~min}$ cada e um intervalo de $15 \mathrm{~min}$ para lanche, trazido pelos próprios participantes. As atividades foram planejadas e dirigidas objetivando a estimulação e reorganização ecológica dos défice previamente preservados pelo grupo. As atividades buscaram cumprir um dos seguintes aspectos: i) reabilitação para 
as atividades de vida diária; ii) resgate e valorização das reminiscências e da identidade pessoal; iii) Estimulação cognitiva e das habilidades sensóriomotoras. (AMERICAN..., 1994). Assim, realizamos técnicas de orientação para a realidade (calendário, relógio, datas comemorativas), recursos mnemônicos (oficina da memória, oficina de jogos, oficina da palavra, bingo), reminiscências (caixa da memória, oficina do jornal), atividades de vida diária (oficina da beleza, oficina do corpo e oficina de expressão) e atividades de socialização (aniversariantes do mês, passeios). (BOTTINO et al., 2002). Além das oficinas descritas, outras atividades são realizadas no projeto, visto que sua aplicabilidade se dá de acordo com o grau de comprometimento dos idosos participantes, aliada às perspectivas sócio-históricas. Assim, a contínua avaliação do quadro demencial e do sujeito e seu contexto é imprescindível no planejamento das oficinas.

Chamamos a atenção para pontos fundamentais que devem ser considerados pelos profissionais ao realizarem as oficinas: tratar a pessoa como um adulto; estar atento a tudo o que acontece ao redor; deixar as pessoas agirem da forma mais independente possível; dar instruções simples (sempre uma por vez); estabelecer uma rotina familiar; falar com a pessoa como se ela fosse capaz de entender; demonstrar sentimentos, ser flexível, criativa; apreender o máximo que puder do passado dessas pessoas e, principalmente, ser paciente. Da mesma forma, o contraponto do que não se deve fazer: falar com a pessoa como se ela fosse uma criança; repreendê-los; falar frases negativas; achar que todas as pessoas com demência são iguais; ter medo de tocá-los ou abraçá-los; falar com eles como se não estivessem lá ou fossem incapazes de entender, sem lhes dar importância, e, por último, desistir. (ALZHEIMER'S..., 1995).

\section{Grupos de orientação aos familiares/cuidadores}

Realizamos, concomitantemente, as oficinas, o trabalho em grupo com os cuidadores, desenvolvendo alternadamente questões mais informativas sobre aspectos do cuidador do idoso com demência, seguindo uma planilha de temas que os próprios cuidadores desejaram conhecer. Além disso, trabalhamos dinâmicas de grupo apropriadas para o reforço positivo do suporte e apoio para bem-estar do cuidador. (SÁ et al., 2006).

\section{Análise estatística}

O experimento realizado é um estudo longitudinal; logo, as amostras não são independentes e todas as avaliações são pareadas. Tendo em vista que os valores de escores dos testes cognitivos não representam variáveis verdadeiramente quantitativas, foram utilizados testes não paramétricos para mensurações repetidas. O teste utilizado para comparar as quatro avaliações (Pré, Pós-1, Pós-2 e Pós-3) foi o teste de Friedman. Quando o teste de Friedman apontava diferenças significativas, era feito o teste dos Postos Sinalizados de Wilcoxon para identificar qual das avaliações se distinguia das demais. As diferenças foram apontadas 
como significativas apenas até o nível de significância de 5\%. O software utilizado foi o R, versão 2.4.1.

\section{Aspectos éticos}

A pesquisa conta com o parecer de aprovado pelo Comitê de Ética em Pesquisa do Centro de Ciências Médicas do Hospital Universitário Antônio Pedro da Universidade Federal. Fluminense, com o protocolo $\mathrm{n}^{\mathrm{a}} 182 / 2005$, de acordo com a resolução $n^{\circ}$ 196/96 do Conselho Nacional de Saúde, cabendo aos pesquisadores o cumprimento dos aspectos éticos com os sujeitos participantes.

\section{Resultados}

A análise dos resultados e sua descrição deram-se a partir da participação nas oficinas e da obtenção dos testes aplicados aos 11 idosos participantes da pesquisa. Assim, iniciamos a apresentação dos dados com a caracterização da clientela atendida.

\section{Caracterização dos sujeitos}

Na Tabela 1 apresentamos os dados de idade, sexo, escolaridade que podem se relacionar com os fatores de risco presentes na demência. O tempo da doença e as patologias associadas podem determinar a gravidade do curso da mesma. O parentesco do cuidador evidência 0 cuidado prestado pela família aos idosos frequentadores do programa.

Tabela 1 - Distribuição de características dos idosos.

\begin{tabular}{|c|c|c|c|c|c|c|}
\hline Idoso & Idade & Sexo & Escolaridade & $\begin{array}{l}\text { Tempo da } \\
\text { doença }\end{array}$ & $\begin{array}{l}\text { Patologias } \\
\text { associadas }\end{array}$ & $\begin{array}{l}\text { Parentesco do } \\
\text { cuidador }\end{array}$ \\
\hline 1 & 87 & $F$ & $4^{a}$ série EF & 2 anos & DM & Formal \\
\hline 2 & 77 & $\mathrm{~F}$ & $3^{\mathrm{a}}$ série $\mathrm{EF}$ & 2 anos & - & Filha \\
\hline 3 & 81 & $M$ & EM & 3 anos & - & Cônjuge \\
\hline 4 & 79 & $\mathrm{~F}$ & $3^{\mathrm{a}}$ série $\mathrm{EF}$ & 2 anos & DM/HAS & Filha \\
\hline 5 & 79 & $\mathrm{~F}$ & $4^{\mathrm{a}}$ série EF & 1 ano & HAS & Filha \\
\hline 6 & 86 & $\mathrm{~F}$ & EM & 6 anos & HAS & Filha \\
\hline 7 & 68 & $\mathrm{~F}$ & $3^{\text {a }}$ série EF & 2 anos & - & Cônjuge \\
\hline 8 & 70 & $\mathrm{~F}$ & $2^{a}$ série EF & 2 anos & DM & Filha \\
\hline 9 & 69 & $\mathrm{~F}$ & Analfabeta & 1 ano & HAS & Filho \\
\hline 10 & 79 & $\mathrm{~F}$ & $4^{\mathrm{a}}$ série EF & 3 anos & HAS & Formal \\
\hline 11 & 76 & $M$ & $4^{\mathrm{a}}$ série EF & 7 anos & - & Cônjuge \\
\hline
\end{tabular}

Nota: F - Feminino; M - Masculino; EF - Ensino fundamental; EM - Ensino médio; DM - Diabetes Mellitus; HAS Hipertensão arterial sistêmica. 
Em relação ao sexo, concluiu-se que a maioria dos participantes (nove) é do sexo feminino e dois do sexo masculino, fato esse associado ao processo de feminilização da velhice. (BOTTINO; LAKS; BLAY, 2006). Já a faixa etária estende-se entre os 67 e 87 anos, com predomínio entre os 76 e 81 anos (seis), corroborando estudos sobre o tema. (SÁ, 2002). Quanto à escolaridade, a maioria (oito) apresenta ensino fundamental incompleto; dois possuem ensino médio e há um caso de analfabetismo. Geralmente, o baixo nível educacional é ressaltado como um provável fator ambiental predisponente à doença. Assim, a baixa escolaridade permite ao indivíduo uma menor compensação para o défice cognitivo, ${ }^{2}$ conforme apontado na literatura.

Em relação ao tempo de doença, o quadro demonstra que a maioria (sete) está entre 2 e 4 anos; dois idosos, entre 6 e 7 anos, e 2 apresentaram seu início há um ano. Em relação a outras patologias associadas, cinco indivíduos apresentam hipertensão arterial sistêmica (HAS); três, diabetes mellitus (DM), e quatro não apresentaram nenhuma outra patologia; entre os participantes um apresentou HAS e DM concomitantemente. Vale ressaltar que os dois homens não apresentaram nenhuma patologia associada à demência.

Em relação ao grau de parentesco dos cuidadores, constatou-se que grande parte dos cuidadores são filhas (cinco); três são esposas; um é filho e dois são cuidadores formais. Portanto, corroboramos a literatura com uma maioria de mulheres. (BOTTINO; LAKS; BLAY, 2006, CALDAS, 2000; SÁ, 2005; MENDES, 1998). Nessa mesma linha de investigação, é importante destacar que verificamos aproximações com os estudos pioneiros no Brasil (MENDES, 1998), os quais mostram que as decisões de assumir os cuidados são mais ou menos conscientes e dependentes de alguns fatores, tais como parentesco (esposa ou filha), gênero (em geral, o feminino), proximidade física e proximidade emocional, esses também encontrados no presente trabalho.

Destacamos o fato de que numa minoria dos casos a pessoa responsável pelos cuidados diários com o idoso não é um familiar, mas um profissional contratado para a realização dessas atividades. Assim, o cuidador principal é aquele que tem a total ou a maior responsabilidade pelos cuidados prestados ao idoso dependente no domicílio, geralmente um familiar. (MENDES, 1998). Os cuidadores secundários seriam outros familiares, voluntários e profissionais que prestam atividades complementares. Usa-se a denominação de "cuidador formal" (principal ou secundário) para o profissional contratado (atendente de enfermagem, acompanhante, empregada doméstica, etc.) e de "cuidador informal", usualmente, para os familiares, amigos e voluntários da comunidade. Nos casos apresentados existem apenas dois idosos que possuem cuidadores formais (profissional contratado); e os demais (nove) possuem cuidadores primários informais (familiares). Isso pode se dever às baixas condições financeiras do grupo, que se encontra entre dois a quatro saláriosmínimos, dado levantado por meio da consulta de enfermagem.

\section{Resultados dos testes de rastreio cognitivo}

A Tabela 2 apresenta os resultados dos testes realizados nos 11 pacientes em quatro avaliações (Pré, Pós-1, Pós-2, e Pós-3) de cada um dos testes propostos (MEEM, relógio, evocação de palavras, AIVD-Lawton e AVD-Katz). 
Priscilla Alfradique de Souza et al.

Tabela 2 - Testes de rastreio cognitivos aplicados trimestralmente em 11 idosos com demência.

\begin{tabular}{|c|c|c|c|c|c|c|c|}
\hline Avaliações & Idosos & MEEM & Relógio & Evocação & Katz & Lawton & EDG \\
\hline & 1 & 17 & 2 & 7 & $\mathrm{D}$ & 11 & 2 \\
\hline & 2 & 18 & 1 & 14 & C & 13 & 2 \\
\hline & 3 & 15 & 1 & 7 & B & 15 & 1 \\
\hline & 4 & 13 & 1 & 3 & $\mathrm{~F}$ & 10 & NA \\
\hline & 5 & 12 & 1 & 9 & B & 12 & 2 \\
\hline \multirow[t]{11}{*}{ Pré } & 6 & 8 & 1 & 5 & B & 11 & 3 \\
\hline & 7 & 18 & 3 & 14 & $A$ & 17 & 4 \\
\hline & 8 & 20 & 4 & 9 & $A$ & 11 & 1 \\
\hline & 9 & 19 & 3 & 8 & C & 10 & 4 \\
\hline & 10 & 10 & 2 & 6 & $E$ & 11 & NA \\
\hline & 11 & 21 & 4 & 12 & A & 18 & 3 \\
\hline & 1 & 18 & 1 & 6 & C & 10 & 4 \\
\hline & 2 & 18 & 1 & 10 & C & 13 & 2 \\
\hline & 3 & 12 & 1 & 5 & B & 12 & 5 \\
\hline & 4 & 17 & 1 & 2 & $\mathrm{~F}$ & 10 & NA \\
\hline & 5 & 12 & 1 & 7 & A & 12 & 2 \\
\hline \multirow[t]{11}{*}{ Pró-1 } & 6 & 6 & 1 & 7 & D & 11 & 4 \\
\hline & 7 & 20 & 3 & 12 & A & 17 & 3 \\
\hline & 8 & 21 & 3 & 11 & A & 12 & 1 \\
\hline & 9 & 20 & 3 & 11 & C & 10 & 4 \\
\hline & 10 & 2 & 1 & 2 & $\mathrm{~F}$ & 10 & NA \\
\hline & 11 & 19 & 3 & 12 & A & 6 & 2 \\
\hline & 1 & 18 & 1 & 3 & C & 12 & 2 \\
\hline & 2 & 18 & 1 & 10 & C & 10 & 4 \\
\hline & 3 & 12 & 1 & 7 & B & 13 & 3 \\
\hline & 4 & 17 & 1 & 2 & $\mathrm{~F}$ & 8 & 1 \\
\hline & 5 & 12 & 1 & 7 & A & 13 & 4 \\
\hline \multirow[t]{11}{*}{ Pró-2 } & 6 & 3 & 1 & 3 & D & 8 & NA \\
\hline & 7 & 14 & 1 & 7 & A & 14 & 4 \\
\hline & 8 & 18 & 2 & 7 & $A$ & 12 & 4 \\
\hline & 9 & 18 & 2 & 8 & C & 10 & 3 \\
\hline & 10 & 2 & NA & 1 & $\mathrm{~F}$ & 10 & NA \\
\hline & 11 & 19 & 3 & 12 & A & 16 & 3 \\
\hline & 1 & 17 & 1 & 4 & C & 12 & 2 \\
\hline & 2 & 11 & 1 & 8 & C & 13 & 4 \\
\hline & 3 & 12 & 2 & 7 & $B$ & 14 & 4 \\
\hline & 4 & 16 & 1 & 3 & $\mathrm{~F}$ & 9 & NA \\
\hline & 5 & 12 & 3 & 7 & A & 14 & 4 \\
\hline \multirow[t]{6}{*}{ Pró-3 } & 6 & 1 & 0 & 2 & $E$ & 8 & NA \\
\hline & 7 & 15 & 1 & 8 & A & 14 & 4 \\
\hline & 8 & 14 & 1 & 3 & C & 8 & 3 \\
\hline & 9 & 11 & 0 & 2 & $E$ & 9 & 1 \\
\hline & 10 & 6 & 0 & 0 & D & 9 & NA \\
\hline & 11 & 22 & 1 & 12 & B & 16 & 2 \\
\hline
\end{tabular}

Nota: NA - não aplicável. 
Podemos observar, para o MEEM, que sete participantes mantiveram seus resultados em, pelo menos, duas realizações dos testes, o que sugere a estabilização no quadro da demência. Observou-se ainda que quatro participantes diminuíram os resultados do MEEM em variações máximas de quatro pontos, o que aponta para a manutenção de suas habilidades cognitivas, com baixo défice de perda. Observamos ainda uma diminuição em $72,7 \%$ dos pacientes estudados (oito). Comparando as quatro avaliações pelo teste de Friedman, o pvalor obtido foi igual a 0,084 , ou seja, não há diferença significativa entre as quatro avaliações. Conclui-se, então, que houve estabilização do estado de demência quando os pacientes foram observados pelo teste MEEM.

$\mathrm{O}$ teste do relógio também nos auxilia na identificação do quadro em que se apresenta a doença. Observamos pela Tabela 2 que sete idosos mantiveram os resultados em todos os testes; dois mantiveram seus resultados em dois testes, $\mathrm{e}$ dois diminuíram a pontuação. Com base nesses resultados, é possível concluir que houve estabilização da doença em $82 \%$ dos participantes. A análise estatística confirma tal afirmação. Pelo teste de Friedman pode se concluir que não houve diferença significativa entre as quatro avaliações $(p=0,080)$, o que evidencia a estabilização do quadro demencial dos pacientes no teste do relógio.

O teste de evocação de palavras, que é aplicado conjuntamente aos dois anteriores, também auxilia na identificação do grau de desenvolvimento da síndrome demencial. Os resultados foram mantidos em seis pacientes pelo menos em dois testes, ao passo que cinco participantes tiveram seus resultados diminuídos, em média, 2,8 pontos. Assim, observamos uma diminuição na pontuação na maioria dos casos estudados (90\%), fato que ocorre normalmente em pacientes que apresentam perda de memória de curto prazo e que necessitam de resposta imediata. (BOTTINO; LAKS; BLAY, 2006).

$\mathrm{O}$ teste de Friedman deixa evidente que o resultado das quatro avaliações é estatisticamente diferente $(\mathrm{p}=0,004)$. Os resultados do Teste de Wilcoxon se encontram na Tabela 3. Do ponto de vista estatístico, o estado do paciente não se alterou entre a pré-avaliação e a primeira pós-avaliação $(\mathrm{p}=0,176)$. Contudo, houve uma redução significativa nos escores da segunda avaliação pós $(p=0,026)$, os quais não diferem significativamente dos escores da terceira avaliação pós ( $p=0,111)$. Enfim, não houve estabilização nem desenvolvimento do estado de evocação de palavras, os escores reduziram significativamente da préavaliação para a terceira pós-avaliação.

Tabela 3 - Comparações múltiplas entre as quatro avaliações do teste de evocação de palavras.

\begin{tabular}{cccc}
\hline Avaliação & Pré & Pós-1 & Pós-2 \\
\hline Pós-1 & 0,176 & & \\
Pós-2 & $0,000^{*}$ & $0,026^{*}$ & \\
Pós-3 & $0,006^{*}$ & $0,018^{*}$ & 0,111 \\
\hline
\end{tabular}

\footnotetext{
* p ignificativo para o teste de Wilcoxon.
} 
Com relação à escala de AIVD Lawton, todos os participantes permaneceram dependentes, isto é, com resultados abaixo de 21 pontos, o que é característico de um paciente demenciado, uma vez que são as primeiras alterações apresentadas no quadro. Dentro esses, seis mantiveram a pontuação, um aumentou-a e quatro diminuíram-na. Apesar de os dados de análise simples mostrarem apenas $36,4 \%$ dos pacientes com valores melhorados ou estabilizados, $54,5 \%$ dos pacientes apresentam resultados estabilizados em, pelos menos, três resultados. Pelo teste de Friedman conclui-se que não houve alterações significativas entre as quatro avaliações $(\mathrm{p}=0,208)$.

Na escala de AVD - Katz, a maioria. Dez dos participantes apresentou seus resultados conservados, e apenas um diminuiu-os. Ainda é possível observar que quatro pacientes tiveram pontuação A, apresentando-se, dessa forma, independentes na realização de todas as atividades do cuidado pessoal. Assim, é percebida a importância desse resultado que preserva as atividades de cuidado pessoal. A análise simples aponta que os resultados permaneceram estabilizados ou aumentaram em 72,7\% dos resultados. A análise estatística do teste de Friedman também demonstra que não houve alterações significativas entre as quatro avaliações, concluindo-se que o quadro dos pacientes permaneceu estabilizado $(p=0,547)$. Esse resultado é de suma relevância nos estudos, uma vez que este teste avalia os quadros de maior dependência. Dessa forma, percebe-se que foram preservadas as questões voltadas para o autocuidado e a independência.
Quanto à aplicação da Escala de Depressão Geriátrica (EDG), foi utilizada para excluirmos as possibilidades de a amostra possuir casos de transtornos do humor. Observamos na Tabela 1 índices abaixo do ponto de corte (7), e em alguns casos não foi possível aplicar a escala (NA) coerentemente, em razão das dificuldades cognitivas. Portanto, não se tornou necessário sofrer o tratamento estatístico, mas consideramos um dado importante para a avaliação das perdas cognitivas relacionadas à demência.

\section{Conclusões}

Concluímos com a descrição e análise deste estudo que os idosos que participaram das atividades de estimulação cognitiva, se comparados a literaturas que expõem idosos com síndrome demencial não estimulados, não avançaram na doença ou apresentaram tênues alterações de seus quadros. Esse fato pode ser observado por meio dos escores no quadro cognitivo dos idosos com demência que participaram das oficinas terapêuticas, evidenciados pelo tratamento estatístico dos instrumentos de rastreio aplicados.

Mesmo conhecendo as limitações desse estudo, como o reduzido número da amostra, as dificuldades de assiduidade dos sujeitos, seja pelo surgimento de patologias associadas, ou pela falta de infraestrutura (fornecimento de transporte, medicação, alimentação, e locais adaptados às necessidades dos idosos e seus cuidadores), contamos com peças fundamentais de auxílio, como o apoio de instituições de fomento à pesquisa e, sobretudo, com a solidariedade dos cuidadores que participaram do projeto. 
Diante do exposto, podemos refletir que a estratégia de cuidados - oficinas de estimulação cognitiva - é aplicável ao trabalho de enfermagem que atua na concepção do cuidado ao idoso com demência. Assim, promovemos aos participantes um cuidado que visa ao incremento de sua funcionalidade, autonomia, individualidade, comunicação e estimulação do idoso à execução de tarefas cotidianas. Além disso, por ser um trabalho em grupo, estimula o processo de socialização, permitindo a manutenção de atividades interpessoais e socioculturais, imprescindíveis à valorização de sua autoestima e à manutenção da independência possível.

Cognitive rehabilitation to aging with dementia: a strategy of caring on Gerontology nursing

\section{Abstract}

We objective to describe and to analyze the workshops stimulation efficacy for aging with dementia through the application of cognitive rake basics instruments. For this, we used the quantitative treatment, prospective and half-experimental, applied of 11 aging, during the time of 08/2006 until 07/2007 in the dependences of Geriatric and Gerontology Program, of the Universidade Federal Fluminense (UFF), in Niterói, State of Rio de Janeiro. The results that were gotten demonstrated the characterization of the clients profile and the statistic treatment of the tests longitudinally. We are able to conclude the viability of the use of the strategic in the nurse's work active in gerontology.

Key words: Geriatric nursing. Health of the elderly. Alzheimer disease.

\section{Referências}

AMERICAN PSIQUIATRIC ASSOCIATION. Diagnostic and statistical manual of mental disorders. 4. ed. Washington: American Psiquiatric Association, 1994.

ALZHEIMER'S ASSOCIATION. Activity programming for persons with dementia: a sourcebook. Chicago: [s. e.], 1995.

BOTTINO, C. M. C.; LAKS, J.; BLAY, S. L. Demência e transtornos cognitivos em idosos. Rio de Janeiro: Guanabara Koogan, 2006.

BOTTINO, C. M. C. et al. Reabilitação cognitiva em pacientes com doença de Alzheimer. Arquivos de Neuropsiquiatria, São Paulo, v. 60 , n. 1, p. 70-79, 2002.

CALDAS, C. P. O sentido do ser cuidando de uma pessoa idosa que vivencia um processo demencial. 2000. 211 f. Tese (Doutorado em Enfermagem) - Escola de Enfermagem Anna Nery, Universidade Federal do Rio de Janeiro, Rio de Janeiro, 2000.

COELHO, G. S. O fundamental no cuidado junto ao idoso com Alzheimer: vivências e experiências de familiares cuidadores compartilhadas no diálogo grupal. 2004. 129 f. Dissertação (Mestrado em Enfermagem) - Escola de Enfermagem Anna Nery, Universidade Federal do Rio de Janeiro, Rio de Janeiro, 2004.

GROISMAN, D. Oficinas terapêuticas para idosos com demência. In: COSTA, C. M.; FIGUEIREDO, A. C. (Org.). Oficinas terapêuticas em saúde mental: sujeito, produção e cidadania. Rio de Janeiro: Coleções IPUB, 2004. p. 247-259.

MENDES, P. B. B. T. Cuidadores: heróis anônimos do cotidiano. In: KARSCH, U. M. S. Envelhecimento com dependência revelando cuidadores. São Paulo: Educ, 1998. p. 171-198.

MENDOZA, R. P. El fantasma del Alzheimer. Temas Enfermagem Actual, v. 6, n. 29, p. 9-11, 1998. 
MUÑOZ, L. A. G.; ARANCIBIA, P. S.; PAREDES, L. P. A. La experiencia de familiares cuidadores de pacientes que sufren alzheimer y competencias del profesional de enfermería. Investigación y Educación en Enfermería, v. 17, n. 2, p. 35-50, 1999.

PELTZER, M. T.; FERNANDES, M. R. Apoiando a família que cuida de seu familiar idoso com demência. Texto \& Contexto Enfermagem, Florianópolis, v. 6, n. 2, p. 339-44, 1997.

SÁ, S. P. C. et al. Oficinas terapêuticas para cuidadores de idosos com demência: atuação da enfermagem no programa interdisciplinar de geriatria e gerontologia da UFF. Revista Brasileira de Geriatria e Gerontologia, Rio de Janeiro, v. 9, n. 3, formato on-line s. n. de páginas, 2006. Manual de cuidados domiciliares na terceira idade.

SÁ, S. P. C. Idoso: representação social da velhice e as implicações no cuidado de si. 2002. 239 f. Tese (Doutorado em Enfermagem) - Escola de Enfermagem Anna Nery, Universidade Federal do Rio de Janeiro, Rio de Janeiro, 2002.

SANTANA, R. F.; SANTOS, I.; CALDAS, C. P. Cuidando de idosos com demência: um estudo a partir da prática ambulatorial em enfermagem. Revista Brasileira de Enfermagem, Brasília, v. 55, n. 1, p. 44-48, 2005.

SOUZA, R. C.; PEREIRA, M. A. O.; SCATENA, M. C. M. Família e transformação da atenção psiquiátrica: olhares que se (des) encontram. Revista Gaúcha de Enfermagem, Porto Alegre, v. 23, n. 2, p. 68-80, 2002. 